--Supporting Information--

\title{
Dry Pressing Neat Active Materials into Ultrahigh Mass Loading Sandwich Cathodes Enabled by Holey Graphene Scaffold
}

Christian O. Plaza-Rivera, Brandon A. Walker, Nam X. Tran, Rocco P. Viggiano, Donald A. Dornbusch, James J. Wu, John W. Connell, * and Yi Lin*

Email: yi.lin-1@,nasa.gov; john.w.connell@,nasa.gov 

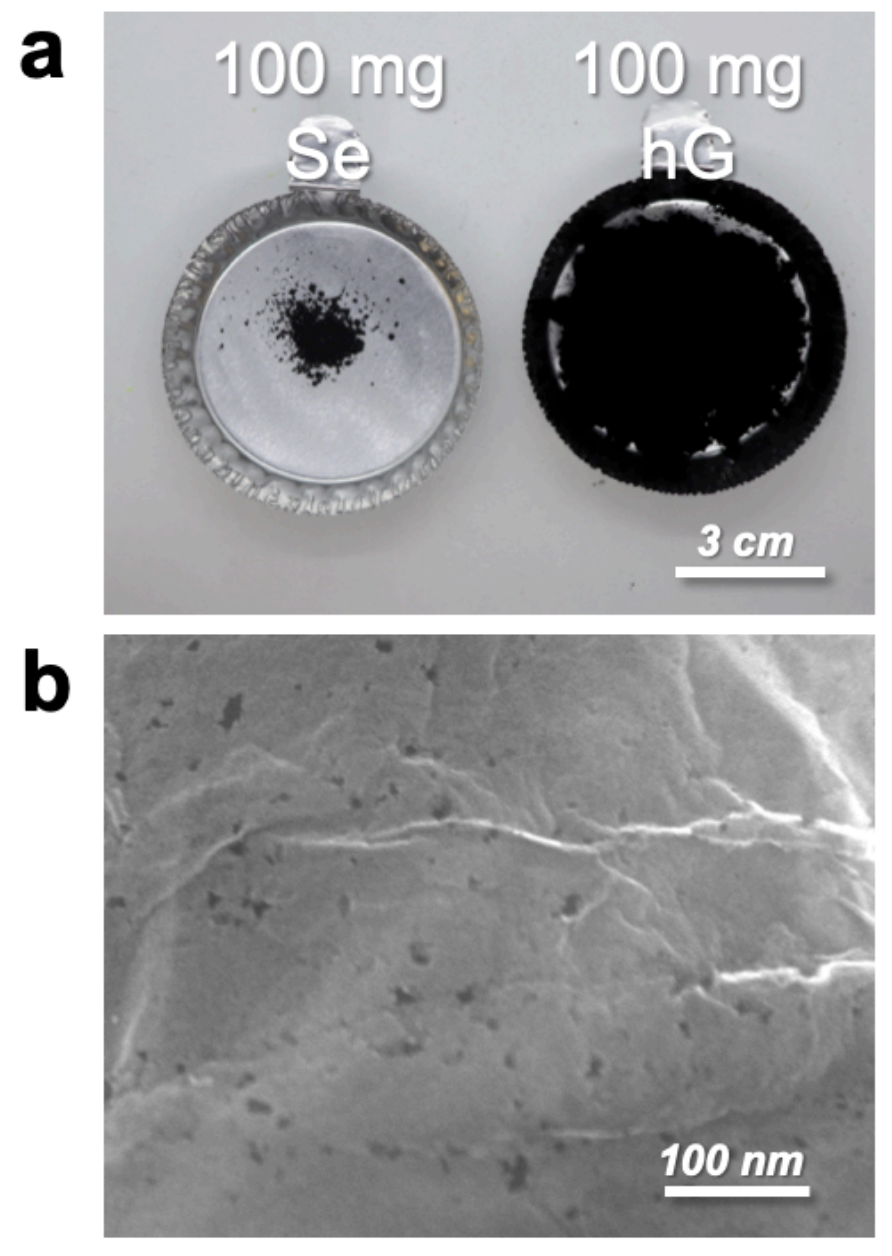

Figure S1. (a) Photograph of $100 \mathrm{mg}$ Se powder and $100 \mathrm{mg} \mathrm{hG}$ powder. (b) A typical SEM image of a hG sheet. 


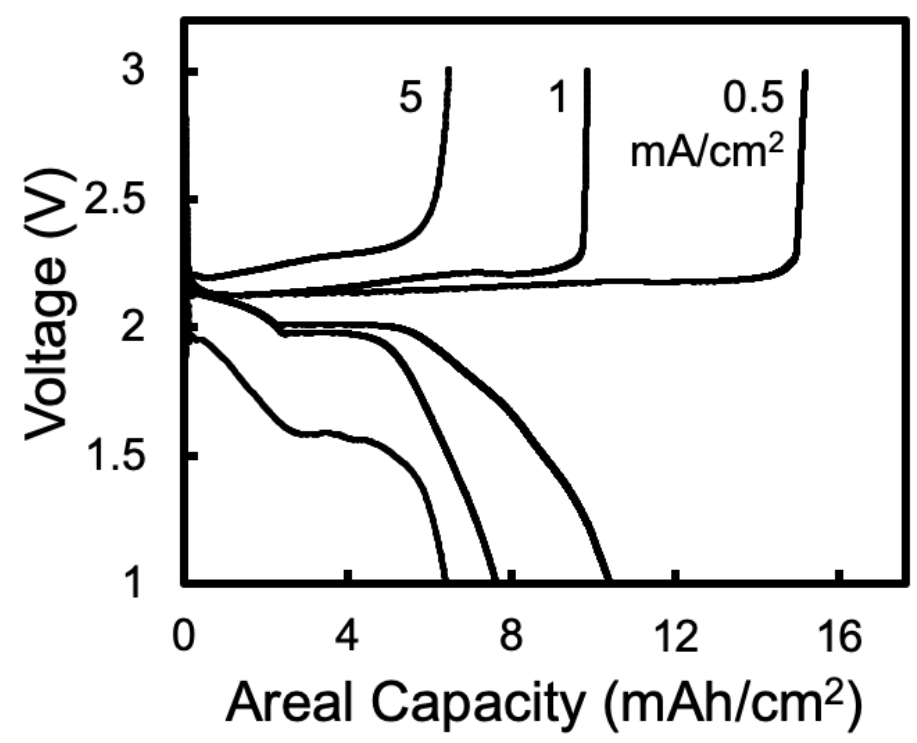

Figure S2. Areal capacity performances from the first discharge and the first charge of three identical Li-Se batteries $\left(m_{\mathrm{Se}}=17.3 \mathrm{mg} / \mathrm{cm}^{2}, \mathrm{Se} \% \sim 75 \mathrm{wt} \%\right)$ ran at $0.5,1$, and $5 \mathrm{~mA} / \mathrm{cm}^{2}$, respectively, in the voltage window of $1-3 \mathrm{~V}$. This is the same set of data shown in Figure 2c but only with the $\mathrm{x}$-axis converted into areal capacity. 

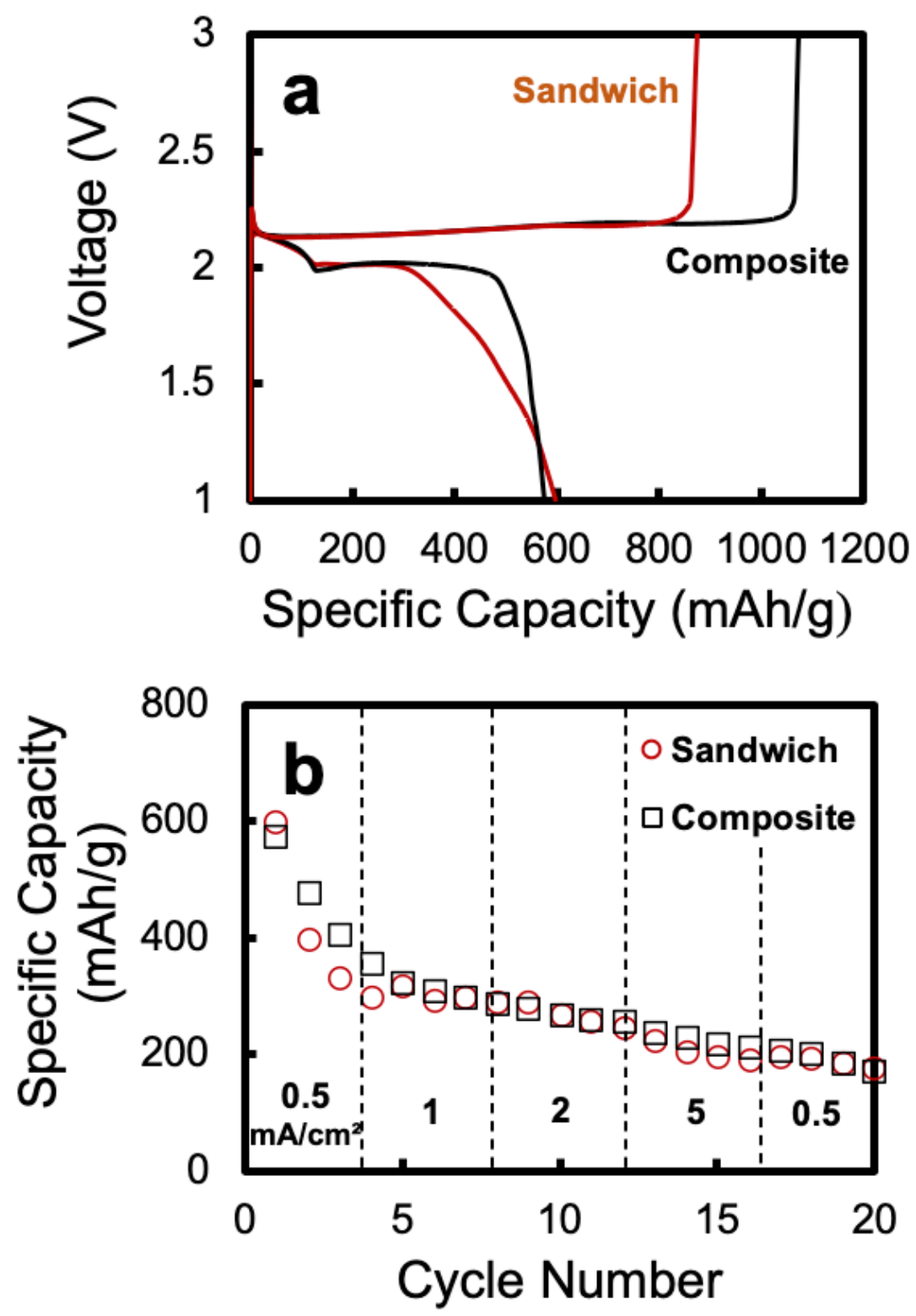

Figure S3. Performance comparison of Li-Se batteries with a $\mathrm{hG} / \mathrm{Se} / \mathrm{hG}$ sandwich cathode vs a hG/Se composite cathode where hG and Se powders were pre-mixed: (a) the first discharge and the first charge curves; (b) specific capacity change at different current densities of $0.5,1,2,5$, and $0.5 \mathrm{~mA} / \mathrm{cm}^{2}$. The total mass of the active material layer were kept the same $(30 \mathrm{mg})$, so that their Se mass loadings and Se contents were slightly different (sandwich: $m_{\mathrm{Se}}=17.3 \mathrm{mg} / \mathrm{cm}^{2}$, $\mathrm{Se} \% \sim 75 \mathrm{wt} \%$; composite: $\left.m_{\mathrm{Se}}=15.6 \mathrm{mg} / \mathrm{cm}^{2}, \mathrm{Se} \% \sim 90 \mathrm{wt} \%\right)$. 
a
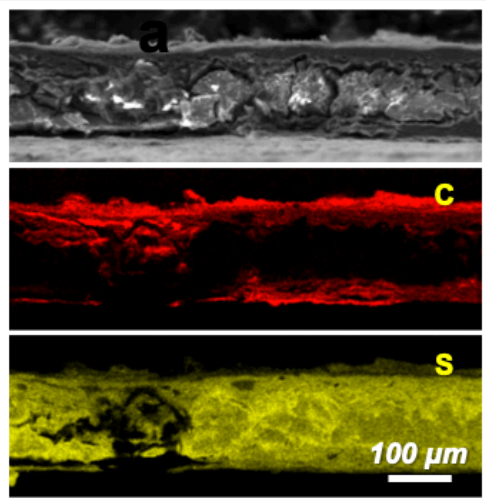

hG/S/hG

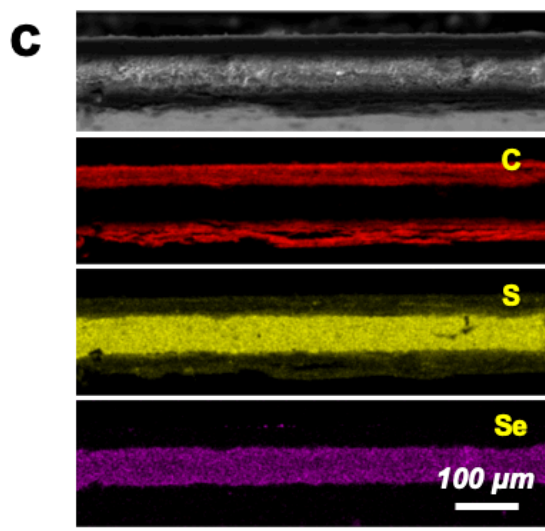

$\mathrm{hG} / \mathrm{SeS}_{2} / \mathrm{hG}$
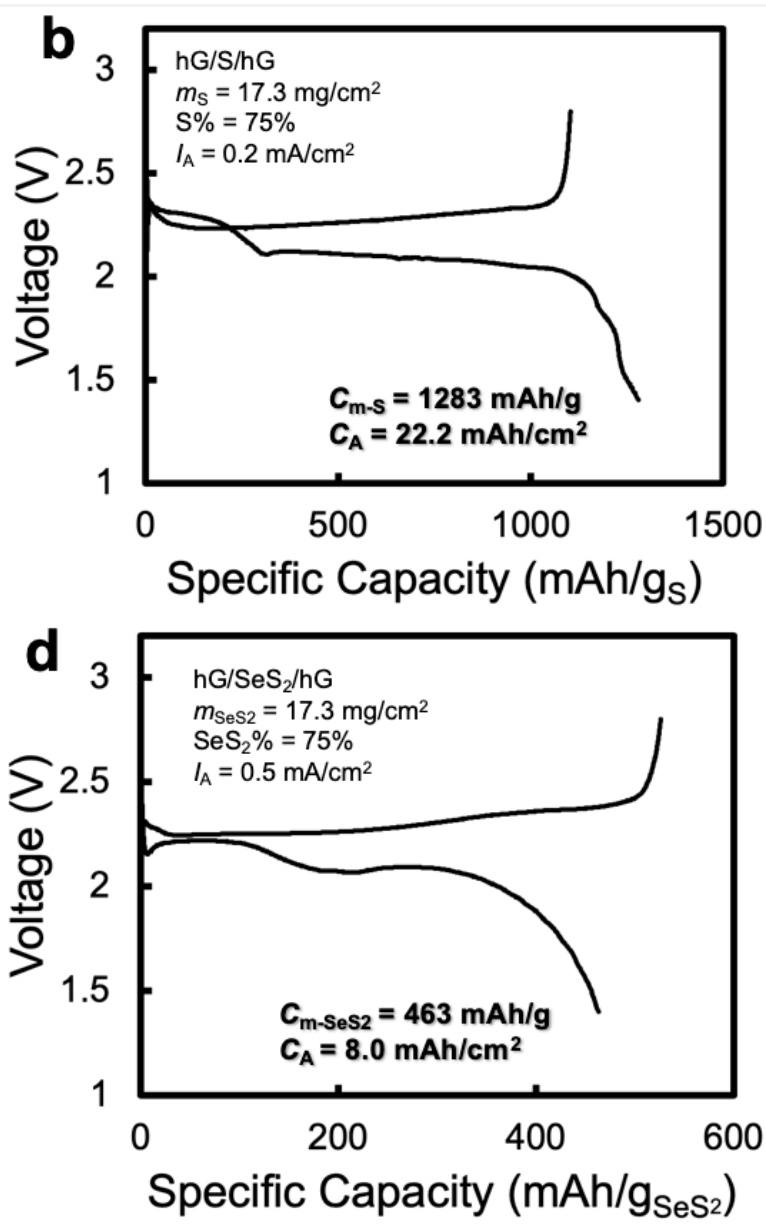

Figure S4. hG-enabled sandwich electrodes with $(\mathrm{a}, \mathrm{b}) \mathrm{S}$ and $(\mathrm{c}, \mathrm{d}) \mathrm{SeS}_{2}$ as the active material, respectively. $(\mathrm{a}, \mathrm{c})$ are the SEM and the corresponding elemental maps of the electrode crosssection. $(b, d)$ are the first discharge and the first charge curves of batteries made with the corresponding sandwich electrode. The electrode characteristics and measurement parameters are specified on each plot. $m_{\mathrm{S}}: \mathrm{S}$ mass loading; $m_{\mathrm{Ses} 2}: \mathrm{SeS}_{2}$ mass loading; $C_{\mathrm{m}-\mathrm{S}}$ and $C_{\mathrm{m}-\mathrm{Ses} 2}$ : specific capacity based on the weight of $\mathrm{S}$ and $\mathrm{SeS}_{2}$, respectively; $C_{\mathrm{A}}$ : areal capacity; $I_{\mathrm{A}}$ : areal current density. 


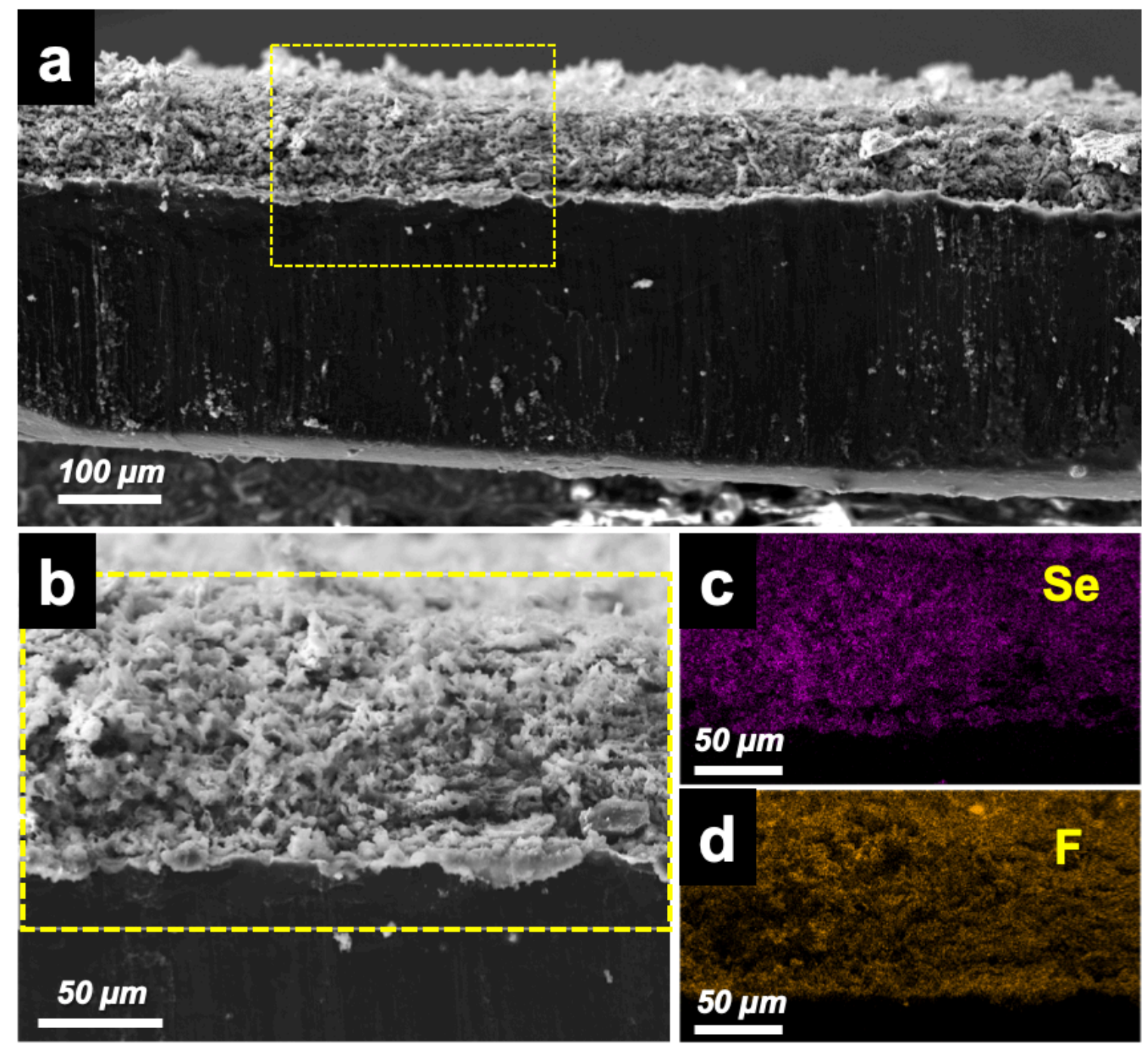

Figure S5. (a) A typical SEM image of cross-section of the Li anode of the Li-Se battery with the $\mathrm{hG} / \mathrm{Se} / \mathrm{hG}$ sandwich cathode $\left(m_{\mathrm{Se}}=17.3 \mathrm{mg} / \mathrm{cm}^{2}, \mathrm{Se} \% \sim 75 \mathrm{wt} \%\right)$ after the first discharge $(1$ D). (b) A magnified view from the area highlighted in (a). (c) Se and (d) F map from EDS analyses of the area highlighted in (b). F element is an indication of SEI as it originated from the electrolyte (LiTFSI). 


\section{Li Anode Separator hG/Se Cathode}

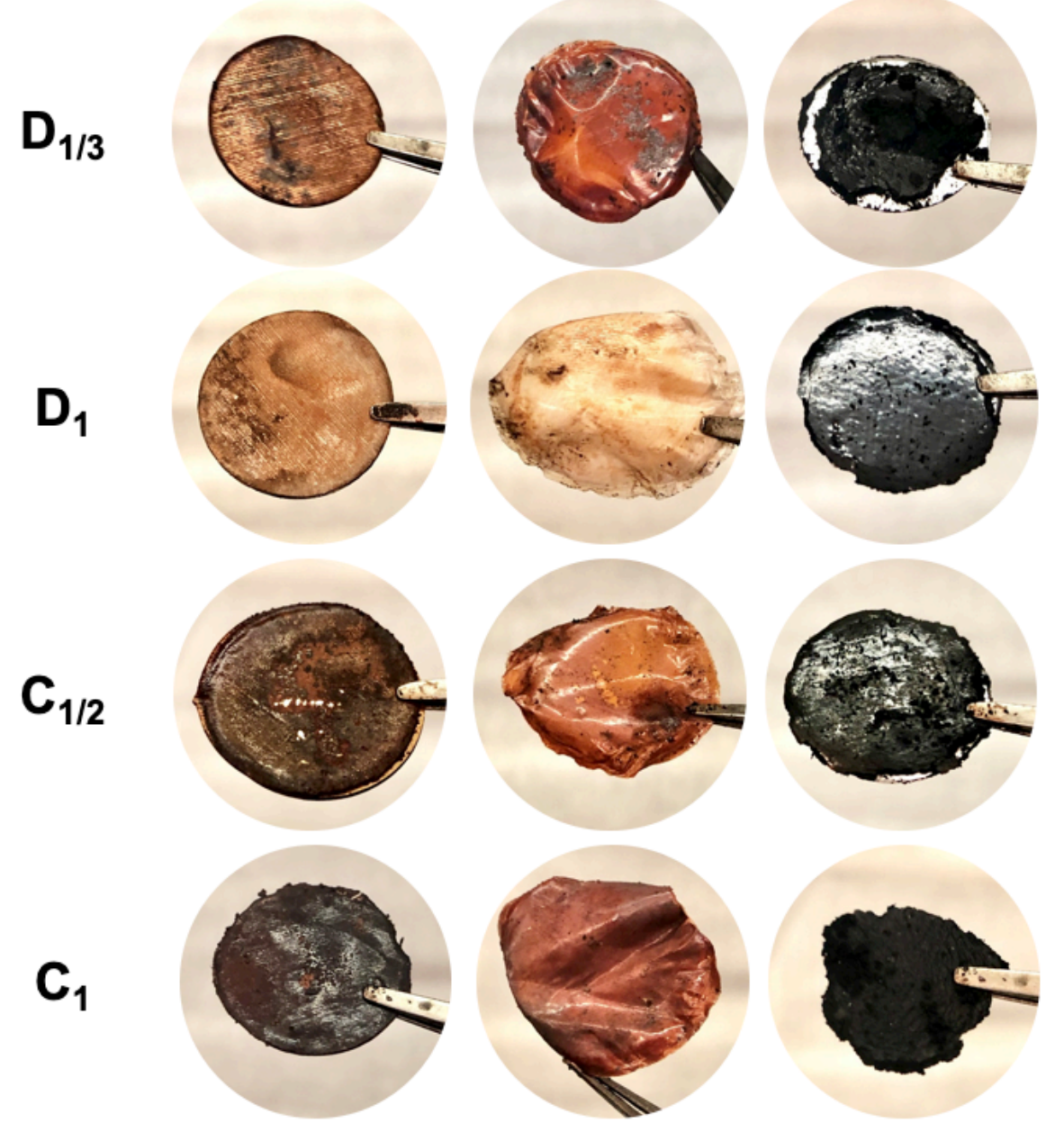

Figure S6. Photographs of post-mortem Li anodes, separators, and the hG/Se cathodes of identical Li-Se batteries with the hG/Se/hG sandwich cathode $\left(m_{\mathrm{Se}}=17.3 \mathrm{mg} / \mathrm{cm}^{2}, \mathrm{Se} \% \sim 75\right.$ $\mathrm{wt} \%)$ that were discharged or charged to different extents at $1 \mathrm{~mA} / \mathrm{cm}^{2}$. 


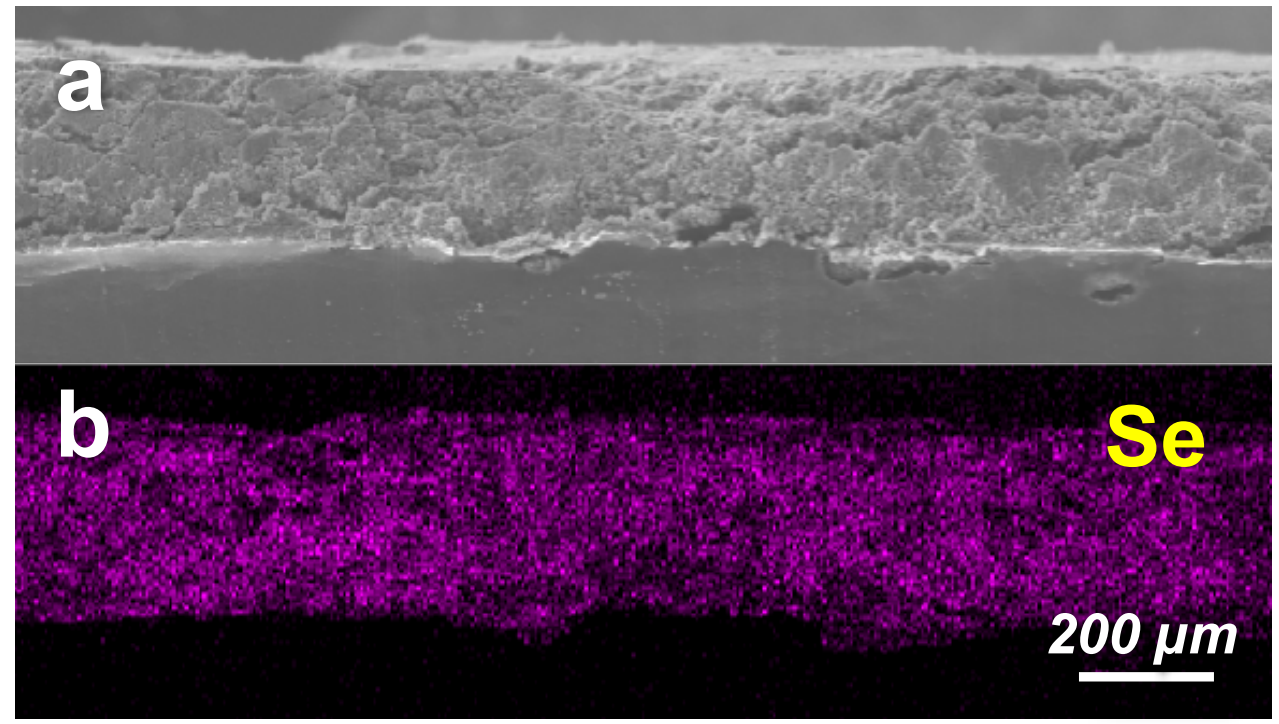

Figure S7. (a) Post-mortem SEM image and (b) the corresponding Se map from EDS mapping of the cathode cross-section, originally in $\mathrm{hG} / \mathrm{Se} / \mathrm{hG}$ sandwich architecture $\left(m_{\mathrm{Se}}=17.3 \mathrm{mg} / \mathrm{cm}^{2}\right.$, $\mathrm{Se} \% \sim 75 \mathrm{wt} \%$ ), from a Li-Se batteries that were run for $>200$ cycles at $1 \mathrm{~mA} / \mathrm{cm}^{2}$. 


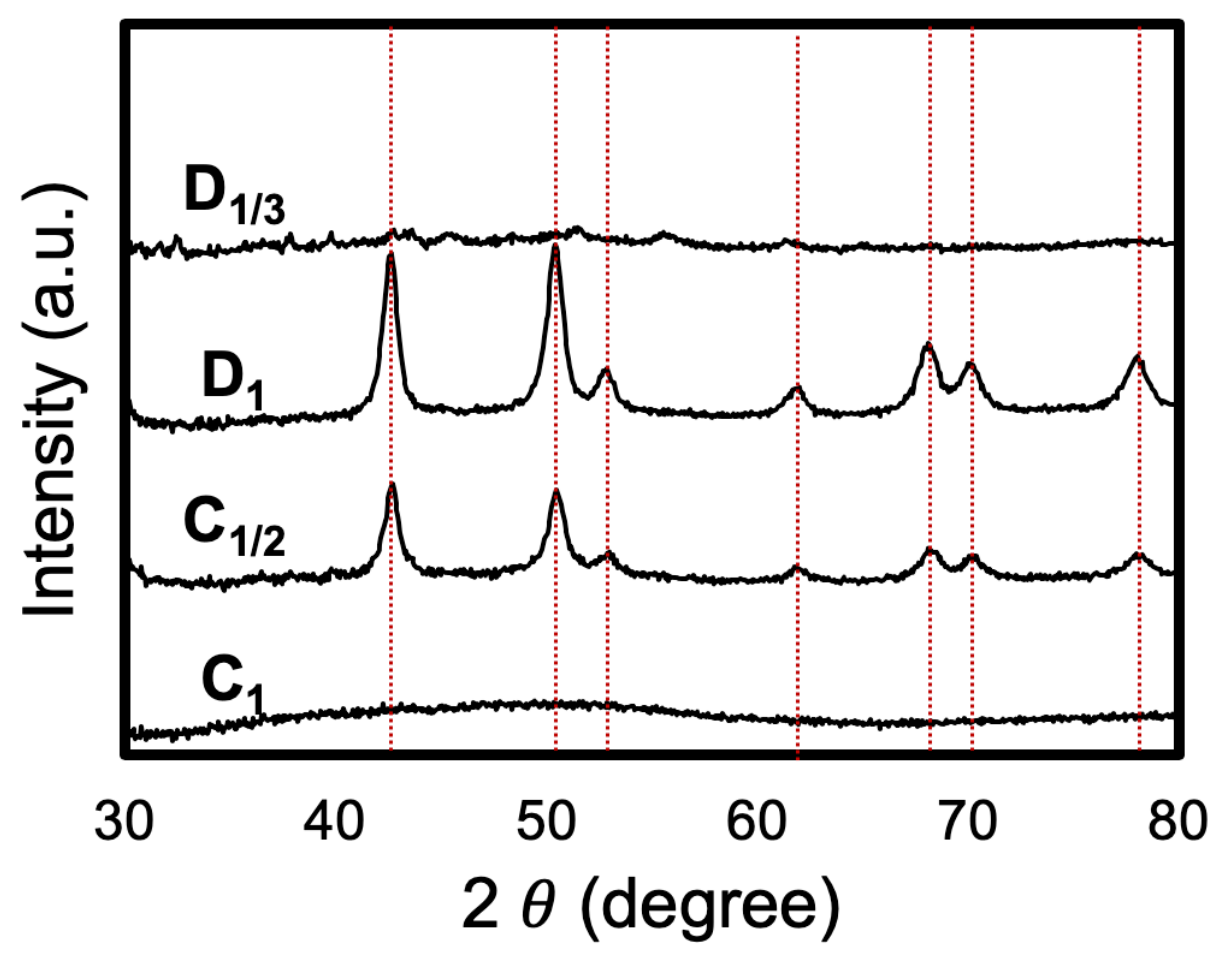

Figure S8. Post-mortem XRD patterns of various cathodes, originally in $\mathrm{hG} / \mathrm{Se} / \mathrm{hG}$ sandwich architecture $\left(m_{\mathrm{Se}}=17.3 \mathrm{mg} / \mathrm{cm}^{2}, \mathrm{Se} \% \sim 75 \mathrm{wt} \%\right)$, from identical Li-Se batteries that were discharged or charged to different extents at $1 \mathrm{~mA} / \mathrm{cm}^{2}$. 


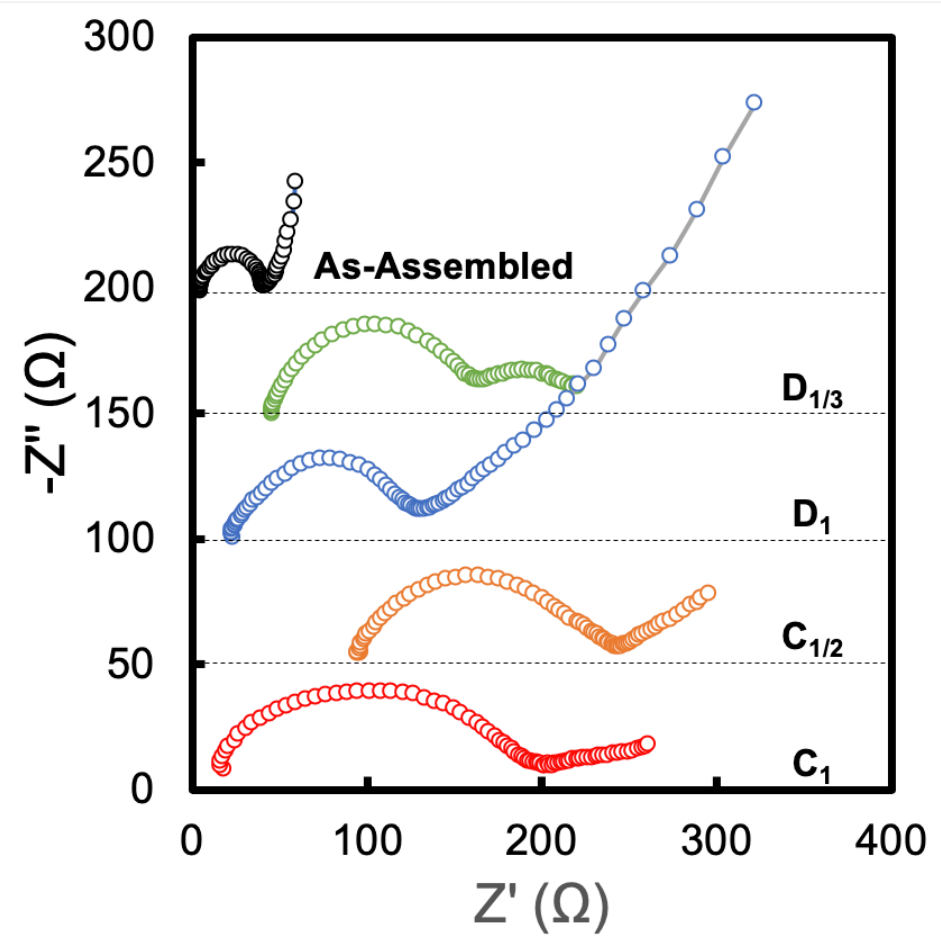

Figure S9. Post-mortem Nyquist plots from EIS measurements of various identical Li-Se batteries with originally $\mathrm{hG} / \mathrm{Se} / \mathrm{hG}$ sandwich cathodes $\left(m_{\mathrm{Se}}=17.3 \mathrm{mg} / \mathrm{cm}^{2}, \mathrm{Se} \% \sim 75 \mathrm{wt} \%\right)$ that were discharged or charged to different extents at $1 \mathrm{~mA} / \mathrm{cm}^{2}$. The Nyquist plot from an asassembled cell (the same shown in Figure $2 \mathrm{a}$ ) is also shown for comparison. 


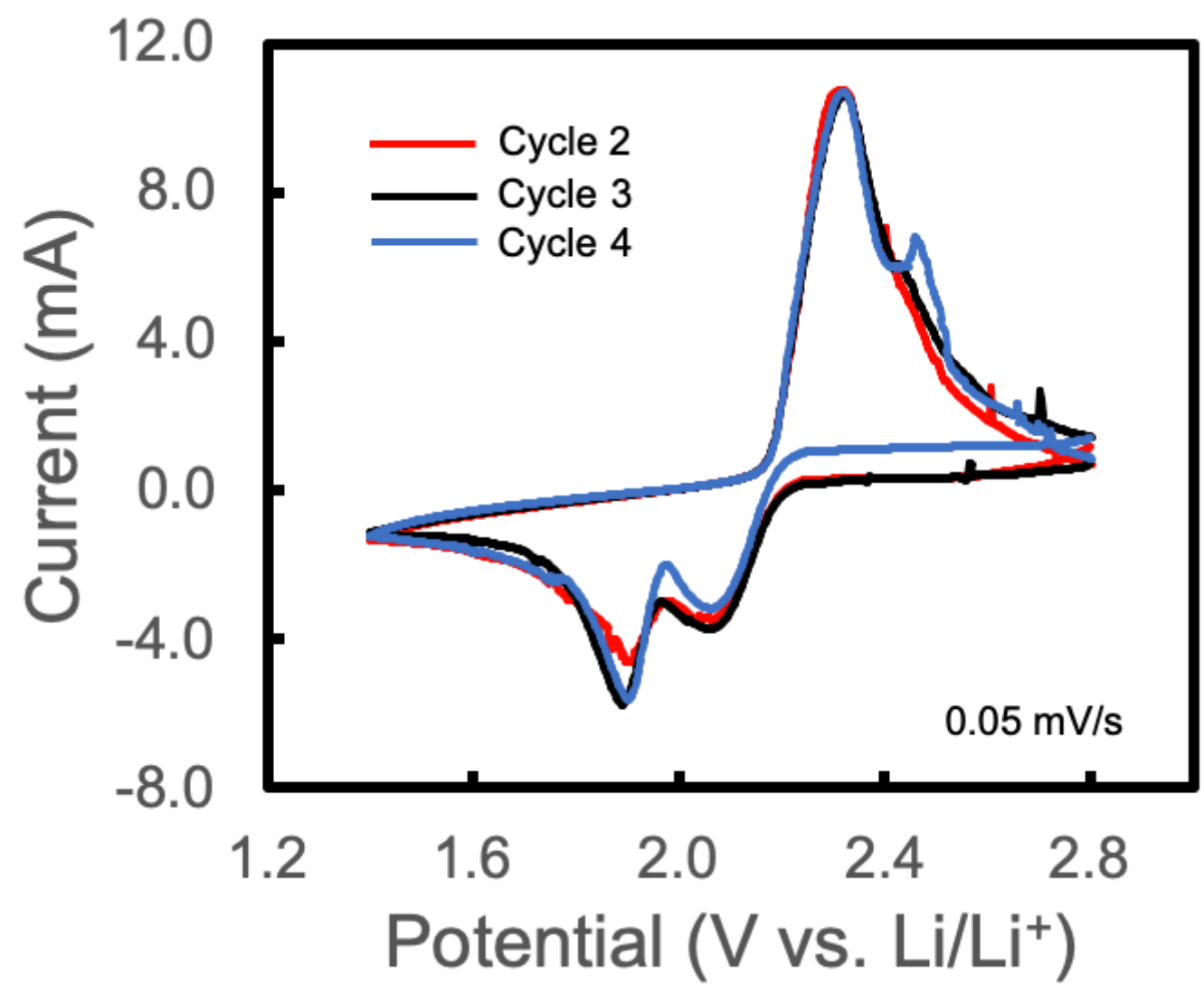

Figure S10. Cycles $2-4$ of the CV measurements for a Li-Se battery with $\mathrm{hG} / \mathrm{Se} / \mathrm{hG}$ sandwich cathode $\left(m_{\mathrm{Se}}=17.3 \mathrm{mg} / \mathrm{cm}^{2}, \mathrm{Se} \% \sim 75 \mathrm{wt} \%\right)$ at a scan rate of $0.05 \mathrm{mV} / \mathrm{s}$ with voltage limits of 1.4 (cathodic) and $2.8 \mathrm{~V}$ (anodic), respectively. These cycles were following Cycle 1 shown in Figure 2b. 


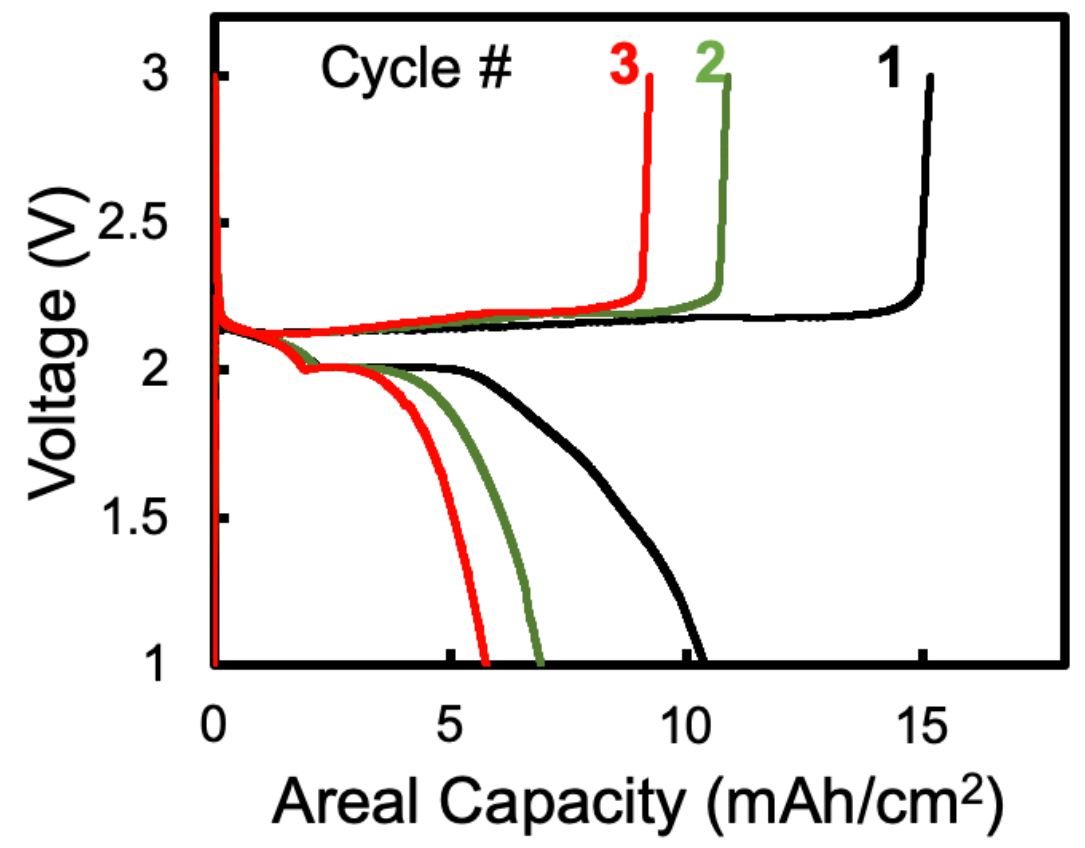

Figure S11. The first 3 cycles of a Li-Se battery with hG/Se/hG cathode $\left(m_{\mathrm{Se}}=17.3 \mathrm{mg} / \mathrm{cm}^{2}\right.$, $\mathrm{Se} \% \sim 75 \mathrm{wt} \%$ ) at a current density of $0.5 \mathrm{~mA} / \mathrm{cm}^{2}$. The cycle 1 data is identical to that shown in Figure 2c. 


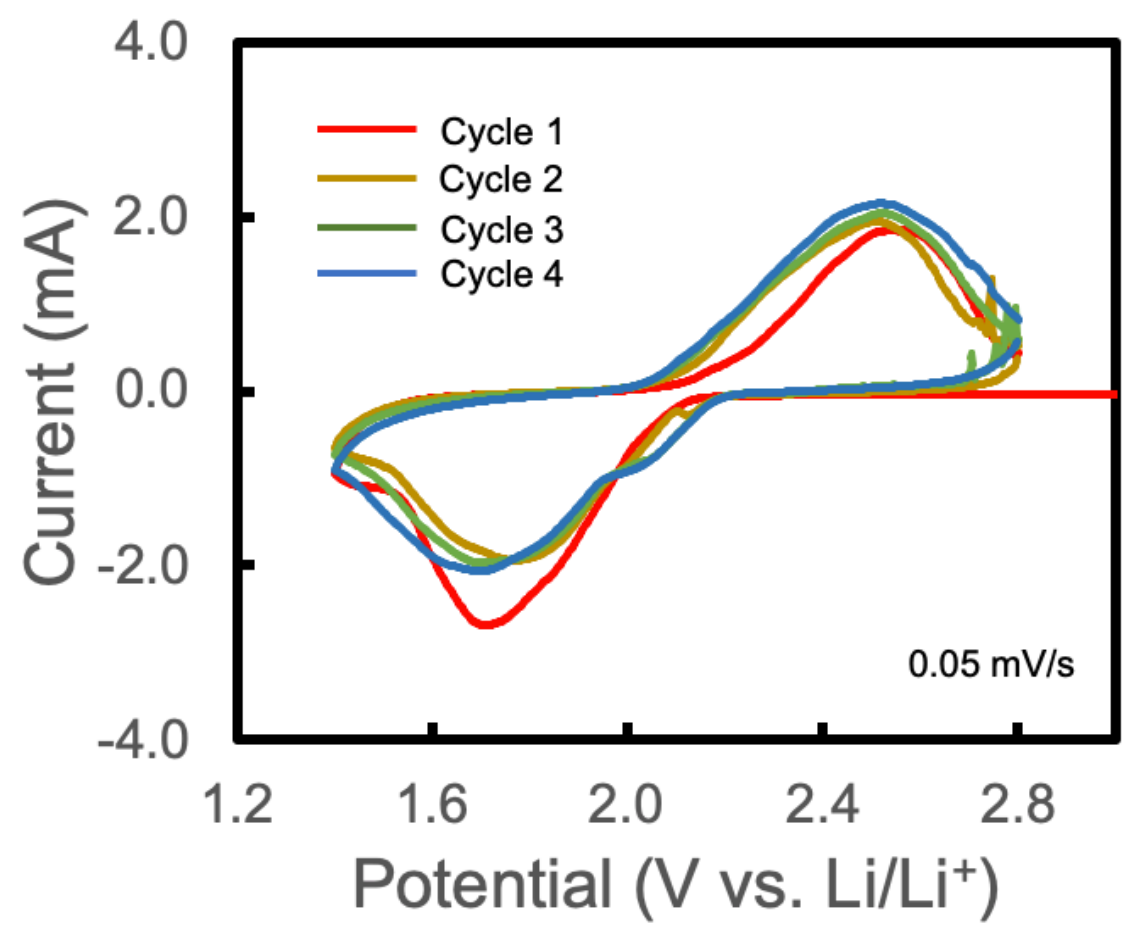

Figure S12. The first 4 cycles of the $\mathrm{CV}$ measurement for a Li-Se battery with $\mathrm{hG} / \mathrm{Se} / \mathrm{hG}$ sandwich cathode $\left(m_{\mathrm{se}}=17.3 \mathrm{mg} / \mathrm{cm}^{2}, \mathrm{Se} \% \sim 75 \mathrm{wt} \%\right)$ and TTE/DOL $(1: 1, \mathrm{v} / \mathrm{v})$ as the electrolyte solvent. The scan rate was $0.05 \mathrm{mV} / \mathrm{s}$; voltage limits were 1.4 (cathodic) and $2.8 \mathrm{~V}$ (anodic), respectively. 

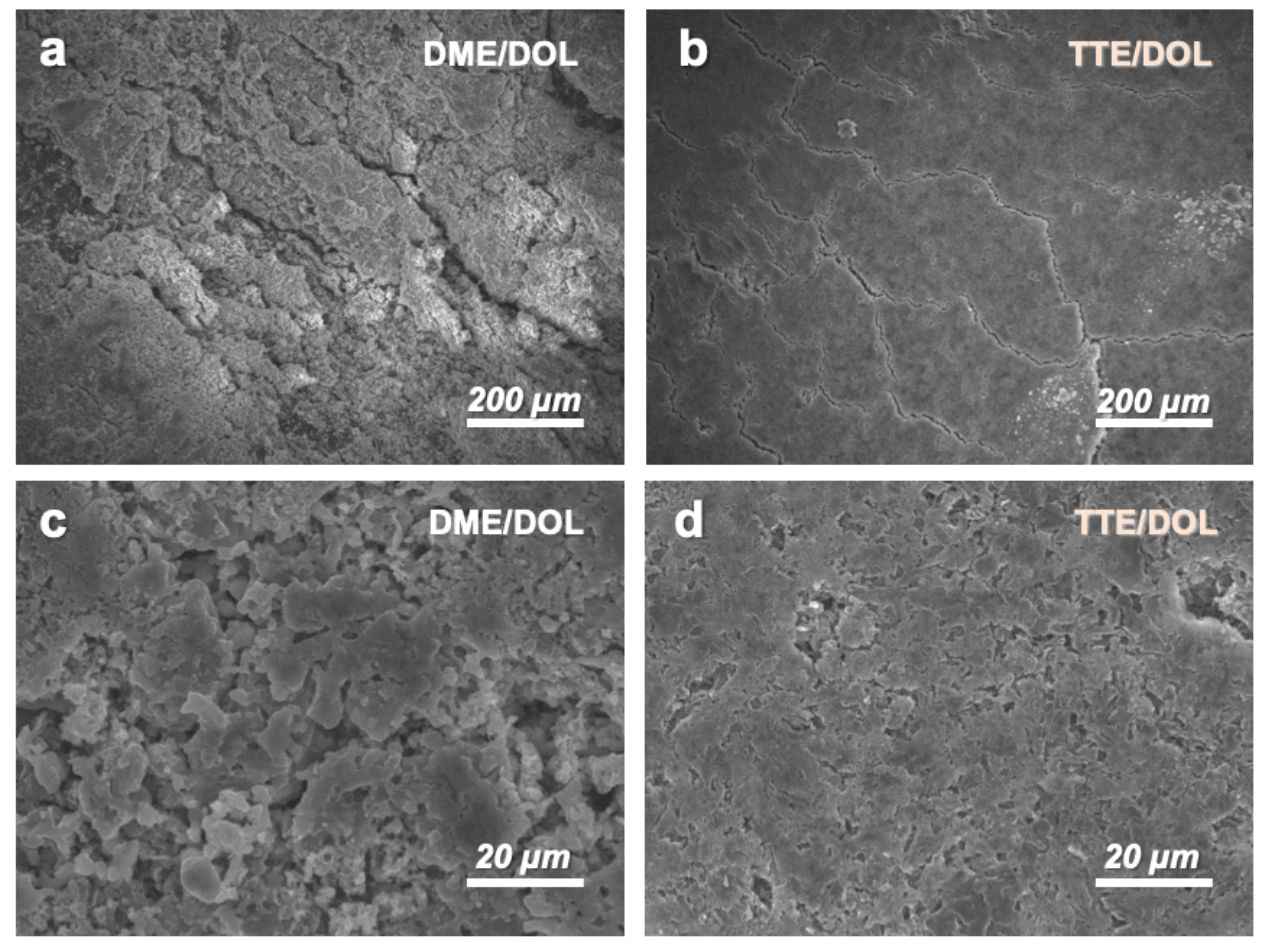

Figure S13. SEM images at $(a, b)$ lower and $(c, d)$ higher magnifications of post-mortem Li anode surfaces of Li-Se batteries with $\mathrm{hG} / \mathrm{Se} / \mathrm{hG}$ sandwich electrodes $\left(m_{\mathrm{Se}}=17.3 \mathrm{mg} / \mathrm{cm}^{2}, \mathrm{Se} \% \sim 75\right.$ wt $\%$ ) after prolonged cycling (> 80 cycles). The electrolyte solvents were (a,c) DME/DOL $(1: 1$, $\mathrm{v} / \mathrm{v})$ and $(\mathrm{b}, \mathrm{d})$ TTE/DOL $(1: 1, \mathrm{v} / \mathrm{v})$, respectively. 
Table S1. Overall and layer density values $\left(\mathrm{g} / \mathrm{cm}^{3}\right)$ of the sandwich discs measured via SEM. Density values of the neat active materials provided by the vendor (Sigma-Aldrich) are also listed for comparison.

\begin{tabular}{l|ccc}
\hline & $\mathrm{hG} / \mathrm{Se} / \mathrm{hG}$ & $\mathrm{hG} / \mathrm{S} / \mathrm{hG}$ & $\mathrm{hG} / \mathrm{SeS}_{2} / \mathrm{hG}$ \\
\hline Overall & 2.31 & 1.46 & 2.09 \\
hG Layer & 1.09 & 0.81 & 0.97 \\
Active Material Layer & 3.68 & 2 & 3.41 \\
Neat Active Material & 4.81 & 2.07 & $\mathrm{~N} / \mathrm{A}$ \\
\hline
\end{tabular}


Table S2. A literature survey (ordered roughly by date of publication) of available capacity values of various Se composite cathodes with Se mass loadings of $>3 \mathrm{mg} / \mathrm{cm}^{2}$ in comparison to those in this work. Blue-shaded entries are those with high Se mass loadings of $>10 \mathrm{mg} / \mathrm{cm}^{2}$.

\begin{tabular}{|c|c|c|c|c|c|c|c|c|c|}
\hline $\begin{array}{l}\text { Cathode } \\
\text { Scaffold }\end{array}$ & $\begin{array}{c}\text { Se } \\
\text { Incorporation } \\
\text { Method }\end{array}$ & $\begin{array}{l}\text { Se Cathode } \\
\text { Preparation }\end{array}$ & $\begin{array}{c}\text { Se } \\
\text { Content } \\
(\%)\end{array}$ & $\begin{array}{l}\text { Se Mass } \\
\text { Loading } \\
\left(\mathrm{mg} / \mathrm{cm}^{2}\right)\end{array}$ & $\begin{array}{c}\text { Current } \\
\text { Density } \\
\text { (mA/cm2) } \\
\text { or } \\
\text { [C-Rate] }\end{array}$ & $\begin{array}{c}\text { Specific Capacity } \\
\text { (mAh/gse) }\end{array}$ & $\begin{array}{c}\text { Areal Capacity } \\
\left(\mathrm{mAh} / \mathrm{cm}^{2}\right)\end{array}$ & $\begin{array}{c}\text { Electrolyte } \\
\text { Base }\end{array}$ & Ref. \\
\hline \multirow{2}{*}{$\begin{array}{l}\text { Holey } \\
\text { Graphene }\end{array}$} & \multirow{2}{*}{$\begin{array}{c}\text { Physical } \\
\text { Sandwich with } \\
\text { a Neat Layer }\end{array}$} & \multirow{2}{*}{ Dry Press } & \multirow{2}{*}{75} & \multirow{2}{*}{17.3} & $0.5 / 1 / 2 / 5$ & $598.4 / 316.2 / 289.1 / 221.3$ & $10.35 / 5.47 / 5.0 / 3.83$ & $\begin{array}{c}\text { Ether } \\
\text { (DME/DOL) }\end{array}$ & \multirow{2}{*}{$\begin{array}{l}\text { This } \\
\text { Work }\end{array}$} \\
\hline & & & & & $0.5 / 1 / 2 / 5$ & 466.7/288.6/154.3/38.10/ & $8.07 / 4.99 / 2.67 / 0.66$ & $\begin{array}{l}\text { Fluorinated } \\
\text { Ether } \\
\text { (TTE/DOL) }\end{array}$ & \\
\hline \multirow{2}{*}{ 3DPSe } & \multirow{2}{*}{ Melt-Diffusion } & \multirow{2}{*}{ 3D Printed } & \multirow{2}{*}{48.2} & 4 & \multirow{2}{*}{$\begin{array}{l}0.5 / 1 / 2 / \\
3 / 5 / 8 / 10\end{array}$} & $\begin{array}{c}657.7 / 568.3 / 531 / 512.4 / \\
485.1 / 441.6 / 401.9\end{array}$ & $2.63 / 2.27 / 2.12 / 2.05 / 1.94 / 1.77 / 1.61$ & \multirow{2}{*}{$\begin{array}{l}\text { Gel- } \\
\text { Polymer }\end{array}$} & \multirow{2}{*}{ [S1] } \\
\hline & & & & 20 & & $580 / 540 / 500 / 470 / 420 / 330 / 270$ & $11.6 / 10.8 / 10 / 9.4 / 8.7 / 6.6 / 5.4$ & & \\
\hline $\begin{array}{l}\text { Covalent } \\
\text { Organic } \\
\text { Framework }\end{array}$ & $\begin{array}{l}\text { High-Speed } \\
\text { Dispersion }\end{array}$ & $\begin{array}{l}\text { Slurry } \\
\text { Casting }\end{array}$ & 80 & $\sim 3$ & {$[6 \mathrm{C} / 20 \mathrm{C}]$} & $126 / 51$ & $0.37 / 0.153$ & $\begin{array}{c}\text { Ether } \\
\text { (DME/DOL) }\end{array}$ & [S2] \\
\hline \multirow{2}{*}{$\begin{array}{l}\text { Multilayer } \\
\text { CNF }\end{array}$} & \multirow{2}{*}{$\begin{array}{l}\text { Syringe- } \\
\text { Filtration }\end{array}$} & \multirow{2}{*}{$\begin{array}{l}\text { Syringe- } \\
\text { Filtration }\end{array}$} & \multirow{2}{*}{63.5} & $4.5 / 6.75$ & $0.22 / 0.34$ & $795 / 818$ & $3.6 / 5.5$ & \multirow{2}{*}{$\begin{array}{c}\text { Ether } \\
\text { (DME/DOL) }\end{array}$} & \multirow{2}{*}{ [S3] } \\
\hline & & & & $9.0 / 13.5$ & $0.45 / 0.675$ & $722 / 667$ & $6.5 / 9.0$ & & \\
\hline \multirow[t]{2}{*}{ ARC-SiOC } & \multirow[t]{2}{*}{ Melt-Diffusion } & \multirow{2}{*}{$\begin{array}{l}\text { Slurry } \\
\text { Casting }\end{array}$} & \multirow[t]{2}{*}{51.2} & $4 / 6$ & \multirow[t]{2}{*}[0.1\mathrm{C}]{} & $850 / 616.6$ & $3.4 / 3.7$ & \multirow[t]{2}{*}{ Carbonate } & \multirow[t]{2}{*}{ [S4] } \\
\hline & & & & $8 / 10$ & & $600 / 350$ & $4.8 / 3.5$ & & \\
\hline $\begin{array}{l}\text { A4-Carbon } \\
\text { Microspheres }\end{array}$ & Melt-Diffusion & $\begin{array}{l}\text { Slurry } \\
\text { Casting }\end{array}$ & 60 & 3.2 & 1.6 & 513 & 1.64 & Carbonate & [S5] \\
\hline $\begin{array}{l}\text { N,S Co- } \\
\text { Doped } \\
\text { Graphene } \\
\text { Membrane }\end{array}$ & Solution Mix & Catholyte & $\mathrm{N} / \mathrm{A}$ & $\sim 5$ & $\begin{array}{c}{[0.5 \mathrm{C} / 1 \mathrm{C} /} \\
2 \mathrm{C} / 4 \mathrm{C}]\end{array}$ & $534.6 / 473.5 / 348.6 / 301.4$ & $2.673 / 2.367 / 1.743 / 1.507$ & $\begin{array}{l}\mathrm{Li}_{2} \mathrm{Se} 8 \\
\text { Solution }\end{array}$ & {$[\mathrm{S} 6]$} \\
\hline $\begin{array}{c}\text { Dual-Doped } \\
\text { Hierarchical } \\
\text { Porous } \\
\text { Carbon } \\
\text { Matrix }\end{array}$ & Melt-Diffusion & $\begin{array}{l}\text { Slurry } \\
\text { Casting }\end{array}$ & $\sim 48$ & 3 & $\mathrm{~N} / \mathrm{A}$ & 280 & 0.84 & Carbonate & [S7] \\
\hline $\begin{array}{l}\text { PANI Coated } \\
\text { Carbon Black }\end{array}$ & Melt-Diffusion & $\begin{array}{l}\text { Slurry } \\
\text { Casting }\end{array}$ & 51.9 & $2-3$ & $\begin{array}{c}3.4 / 5.06 / \\
8.4 \\
{[2 \mathrm{C} / 3 \mathrm{C} / 5 \mathrm{C}]}\end{array}$ & $529.2 / 514.2 / 475.6$ & 1.3/1.29/1.19 & Carbonate & [S8] \\
\hline $\begin{array}{l}\text { Nano- } \\
\text { Cellulose } \\
\text { Derived } \\
\text { Monolithic } \\
\text { Carbon }\end{array}$ & melt-Infiltration & $\begin{array}{l}\text { Slurry } \\
\text { Casting }\end{array}$ & 70 & 6.64 & {$[0.1 \mathrm{C}]$} & 620 & 4.12 & Carbonate & [S9] \\
\hline CNTs & $\begin{array}{l}\text { Pressure- } \\
\text { Induced Melt- } \\
\text { Diffusion }\end{array}$ & $\begin{array}{l}\text { Slurry } \\
\text { Casting }\end{array}$ & 85 & $5-10$ & $\begin{array}{l}0.2 \mathrm{C} / 0.5 \mathrm{C} / \\
1 \mathrm{C} / 2 \mathrm{C}\end{array}$ & $333 / 231 / 153 / 100$ & $2.5 / 1.73 / 1.15 / 0.75$ & $\begin{array}{l}\text { Ether } \\
\text { (DME/DOL) }\end{array}$ & [S10] \\
\hline G-SeHMs & $\begin{array}{l}\text { Graphene } \\
\text { Oxide /Se } \\
\text { suspension }\end{array}$ & $\begin{array}{l}\text { Slurry } \\
\text { Casting }\end{array}$ & 80 & $2-3$ & $\begin{array}{l}0.17 / 8.43 \\
{[0.1 \mathrm{C} / 5 \mathrm{C}]}\end{array}$ & $514 / 241$ & $1.285 / 0.6$ & $\begin{array}{c}\text { Ether } \\
\text { (TEGDME/ } \\
\text { DOL) }\end{array}$ & [S11] \\
\hline $\begin{array}{c}\text { Macro- } \\
\text { /Micro- } \\
\text { Porous } \\
\text { Biochar- } \\
\text { Based } \\
\text { Framework }\end{array}$ & Melt-Diffusion & $\begin{array}{l}\text { Slurry } \\
\text { Casting }\end{array}$ & 56.1 & $2.9 \pm 0.2$ & {$[0.2 \mathrm{C}]$} & 466.8 & 1.35 & Carbonate & [S12] \\
\hline
\end{tabular}




\begin{tabular}{|c|c|c|c|c|c|c|c|c|c|}
\hline $\begin{array}{l}\text { Multi-Walled } \\
\text { Carbon } \\
\text { Nanotube }\end{array}$ & Solution Mix & $\begin{array}{l}\text { Slurry } \\
\text { Casting }\end{array}$ & 60.83 & 3.6 & $\begin{array}{r}1.21 / 2.43 \\
14.86 / 9.72\end{array}$ & $\sim 680 / 580 / 500 / 358.2$ & $2.45 / 2.09 / 1.8 / 1.29$ & $\begin{array}{c}\text { Ether } \\
\text { (DME/DOL) }\end{array}$ & [S13] \\
\hline $\mathrm{Se}$ & -- & $\begin{array}{l}\text { Slurry } \\
\text { Casting }\end{array}$ & 70 & 4 & $2.7[0.5 \mathrm{C}]$ & 546 & 2.18 & $\begin{array}{c}\text { Ether } \\
\text { (DME/DOL) }\end{array}$ & [S14] \\
\hline $\begin{array}{l}\text { Porous } \\
\text { Hollow } \\
\text { Carbon } \\
\text { Bubbles }\end{array}$ & Melt-Diffusion & $\begin{array}{l}\text { Slurry } \\
\text { Casting }\end{array}$ & 48 & $3-4$ & $\begin{array}{c}{[0.1 \mathrm{C} / 0.2 \mathrm{C} /} \\
0.5 \mathrm{C} / 1 \mathrm{C}\end{array}$ & $600 / 525 / 465 / 425$ & $2.1 / 1.84 / 1.63 / 1.49$ & Carbonate & [S15] \\
\hline
\end{tabular}

\section{References:}

[S1] Gao, X.; Yang, X.; Wang, S.; Sun, Q.; Zhao, C.; Li, X.; Liang, J.; Zheng, M.; Zhao, Y.; Wang, J.; Li, M.; Li, R.; Sham, T.-K.; Sun, X. A 3D-printed ultra-high Se loading cathode for high energy density quasi-solid-state Li-Se batteries. J. Mater. Chem. A 2020, 8, 278-286.

[S2] Si, L.; Wang, J.; Li, G.; Hong, X.; Wei, Q.; Yang, Y.; Zhang, M.; Cai, Y. High energy density lithium-selenium batteries enabled by a covalent organic framework-coated separator Mater. Lett. 2019, 246, 144-148.

[S3] Xia, Y.; Lu, C.; Fang, R.; Huang, H.; Gan, Y.; Liang, C.; Zhang, J.; He, X.; Zhang, W. Freestanding layer-structure selenium cathodes with ultrahigh Se loading for high areal capacity Li-Se batteries. Electrochem. Commun. 2019, 99, 16-21.

[S4] Fang, R.; Xia, Y.; Liang, C.; He, X.; Huang, H.; Gan, Y.; Zhang, J.; Tao, X.; Zhang, W. Supercritical $\mathrm{CO}_{2}$-assisted synthesis of $3 \mathrm{D}$ porous $\mathrm{SiOC} / \mathrm{Se}$ cathode for ultrahigh areal capacity and long cycle life Li-Se batteries. J. Mater. Chem. A 2018, 6, 24773-24782.

[S5] Park, G. D.; Kim, J. H.; Lee, J.-K.; Kang, Y. C. Carbon microspheres with well-developed micro- and mesopores as excellent selenium host materials for lithium-selenium batteries with superior performances. J. Mater. Chem. A 2018, 6, 21410-21418.

[S6] Gu, X.; Xin, L.; Li, Y.; Dong, F.; Fu, M.; Hou, Y. Highly reversible Li-Se batteries with ultra-lightweight N,S-codoped graphene blocking layer. Nano-Micro Lett. 2018, 10, 59.

[S7] Zhao, X.; Yin, L.; Zhang, T.; Zhang, M.; Fang, Z.; Wang, C.; Wei, Y.; Chen, G.; Zhang, D.; Sun, Z.; Li, F. Heteroatoms dual-doped hierarchical porous carbon-selenium composite for durable Li-Se and $\mathrm{Na}-\mathrm{Se}$ batteries. Nano Energy 2018, 49, 137.

[S8] Wang, B.; Zhang, J.; Xia, Z.; Fan, M.; Lv, C.; Tian, G.; Li, X. Polyaniline-coated selenium/carbon composites encapsulated in graphene as efficient cathodes for Li-Se batteriesNano Res. 2018, 11, 2460-2469.

[S9] Ding, J.; Zhou, H.; Zhang, H.; Tong, L.; Mitlin, D. Selenium impregnated monolithic carbons as free-standing cathodes for high volumetric energy lithium and sodium metal batteries. Adv. Energy Mater. 2017, 8, 1701918.

[S10] Dutta, D.; Gope, S.; Negi, D. S.; Datta, R.; Sood, A. K.; Bhattacharyya, A. J. Pressureinduced capillary encapsulation protocol for ultrahigh loading of sulfur and selenium inside 
carbon nanotubes: Application as high-performance cathode in $\mathrm{Li}-\mathrm{S} / \mathrm{Se}$ rechargeable batteries. $J$. Phys. Chem. C 2016, 120, 51,29011-29022.

[S11] Youn, H.-C.; Jeong, J. H.; Roh, K. C.; Kim, K.-B. Graphene-selenium hybrid microballs as cathode materials for high-performance lithium-selenium secondary battery applications. Sci. Rep. 2016, 6, 30865.

[S12] Zhang, H.; Yu, F.; Kang, W.; Shen, Q. Encapsulating selenium into macro-/micro-porous biochar-based framework for high-performance lithium-selenium batteries. Carbon 2015, 95, 354-363.

[S13] Wang, X.; Zhang, Z.; Qu, Y.; Wang, G.; Lai, Y.; Li, J. Solution-based synthesis of multiwalled carbon nanotube/selenium composites for high performance lithium-selenium battery. $J$. Power Sources 2015, 287, 247-252.

[S14] Fang, R.; Zhou, G.; Pei, S.; Li, F. Cheng, H.-M. Localized polyselenides in a graphenecoated polymer separator for high rate and ultralong life lithium-selenium batteries. Chem. Commun. 2015, 51, 3667-3670.

[S15] Zhang, J.; Fan, L.; Zhu, Y.; Xu, Y.; Liang, J.; Wei, D.; Qian, Y. Selenium/interconnected porous hollow carbon bubbles composites as the cathodes of $\mathrm{Li}-\mathrm{Se}$ batteries with high performance. Nanoscale 2014, 6, 12952-12957. 